Rechtsmedizin 2020 · 30:311-317 https://doi.org/10.1007/s00194-020-00423-y Online publiziert: 14 . September 2020

(c) Der/die Autor(en) 2020
V. Hofer ${ }^{1}$ J. Galli $\cdot$ M. J. Thali • R. M. Martinez

${ }^{\prime}$ Institut für Rechtsmedizin, Universität Zürich, Zürich, Schweiz

\title{
„Flying Forensic Nursing“ in der Versorgung und Beratung von Gewaltbetroffenen
}

\section{Auswertung erster Erfahrungen mit diesem neuen Konzept}

\begin{abstract}
„Flying Forensic Nursing“ ist ein Projekt in der modernen Versorgung von (gewaltbetroffenen) Personen im forensischen Kontext. Das Projekt öffnet den Auftraggebern (Staatsanwaltschaft und Polizei), aber auch den Beauftragten (z. B. Amtsärzten, Justiz- und Vollzugsdiensten) neue Perspektiven für eine zeitnahe und professionelle Unterstützung bei den Untersuchungen von Gewaltbetroffenen und bei der Befunderhebung als auch bei der Überprüfung einer Fahrunfähigkeit durch eine forensisch spezialisierte Pflegefachperson. Durch das immer dichter werdende Netzwerk von forensisch geschulten Gesundheitsfachpersonen kann das Angebot des Flying Forensic Nursing auch in Regionen mit schwacher forensischer Infrastruktur sichergestellt werden.
\end{abstract}

\section{Hintergrund}

Die Autorin des Projekts „Flying Forensic Nursing" [1] ist als diplomierte Expertin Notfallpflege HF (höhere Fachschule) tätig und blickt auf eine über 32-jährige Berufserfahrung, die sie auf verschiedenen interdisziplinären Notfallstationen in der Schweiz und im Ausland gesammelt hat, zurück. Ihre Erfahrung zeigt, dass Menschen, die von Gewalt betroffen sind, sowie auch Personen, die zwecks Blut- und Urinprobenentnahmen zur Bestimmung eines evtl. Fremdsubstanzkonsums polizeilich zugeführt werden, meist keine oder nur ungenügende professionelle Behandlung erhalten. Die Gründe sind einerseits Zeitmangel und andererseits fehlende Detail- und Prozesskenntnisse des Krankenhauspersonals über die korrekte Vorgehensweise bei der Betreuung der Betroffenen. Dadurch ist die Dokumentation von Befunden fehler- und lückenhaft und deren Aussagekraft in möglichen späteren Straf- und Zivilverfolgungsprozessen eingeschränkt [2]. Aufgabe der vorliegenden Studie war es, diese subjektiven Erkenntnisse, auch wenn diese auf eine sehr erfahrene Fachkraft zurückgehen, durch systematische Untersuchungen zu objektivieren.

In der Projektarbeit wird beschrieben, wie diese Missstände durch den Einsatz einer Flying Forensic Nurse beseitigt werden können. Um eine professionelle Untersuchung mit vollständiger und gerichtsverwertbarer Dokumentation zu erstellen, werden Zeit, Sorgfalt und ein klar definierter generischer Arbeitsprozess benötigt. Dieser beinhaltet sowohl das „Wer", also die Funktion des Untersuchenden, das "Was", die jeweiligen Aufgaben, als auch das „Wie“, die nötigen Arbeitsschritte und Tätigkeiten. Dies trägt wesentlich zur Systematisierung des Fachs bei. Durch eine Definition des Berufsprofils einer Flying Forensic Nurse, einschließlich möglicher Aufgaben und Verantwortlichkeiten, soll dieser Arbeitsprozess konkretisiert werden. Doch die Festlegung der Kompetenzen und der Handlungsfelder einer Flying Forensic Nurse ist durch viele Faktoren erschwert und bedarf einer Klärung und nicht zuletzt spezifischer Erfahrung im interdisziplinären Kontext: Die ersten Implementierungsversuche des 2019 erstellten Kompetenzprofils von Forensic Nurses in der Schweiz fielen in den eigenen rechtsmedizinischen Kreisen durch - die Rückmeldungen zum Kompetenzprofil von Forensic Nurses aus verschiedenen rechtsmedizinischen Instituten der Schweiz ließen darauf schließen, dass die Grundkompetenzen, Verantwortlichkeiten und Handlungsfelder einer Pflegefachperson im klinischen Berufsalltag den Rechtsmedizinern größtenteils nicht oder nur teilweise bekannt sind.

Die Situation in Bezug auf die Kompetenzen und Verantwortlichkeiten einer Pflegefachperson sieht in den Krankenhäusern oder in den Gemeinschaftsarztpraxen hingegen anders aus: Durch die enge Zusammenarbeit zwischen den Ärzten und den Pflegefachpersonen sind die Aufgabenbereiche, Handlungs- und auch Entscheidungsfelder beider Berufsgruppen klar definiert und bilden somit die optimale Grundlage für die professionelle und ressourcenorientierte Patientenversorgung. Galli formulierte im Rahmen ihrer Certificate-of-Advanced-Studies(CAS)-Weiterbildung in Forensic Nursing an der Universität Zürich (UZH) 2017 eine entsprechende Vision: ein "forensic nurse network", das 365 Tage im Jahr und 24h am Tag kompetente und professionelle Leistungen in unterschiedlichen Institutionen (Polizei, Staatsanwaltschaft, Krankenhäuser 
und übrige Antragsteller) anbietet. Dies ist für alle involvierten Personen, die Angehörigen der „Blaulichtorganisationen", jedoch auch besonders für die von Gewalt betroffenen Menschen ein niederschwelliges Angebot zu schneller Hilfe und professioneller Unterstützung [1]. Die vorliegende Arbeit soll durch systematische Untersuchungen die objektiven Parameter für die Umsetzung der Vision in die tägliche Praxis ermitteln.

\section{Methode}

Mithilfe einer systematischen Analyse der Ist-Situation verschiedenster Aspekte sollte die aktuelle Situation der Forensic Nurses in der Schweiz untersucht werden. Hierzu zählen strukturelle Abläufe auf einer Notfallstation, des Schnittstellenmanagements, die Erfüllung forensischer Aufgaben, wie Dokumentation der Befunde und Sicherung biologischer Spuren auf der Notfallstation, sowie der rechtlichen Aspekte, die die Asservatenhandhabung auf der Notfallstation betreffen. $\mathrm{Zu}$ Überprüfung und Objektivierung der persönlichen Erfahrungen einer realen Flying Forensic Nurse wurden weitere Abklärungen unternommen. So erfolgte neben zahlreichen Interviews mit Vertretern von Polizei und Justizbehörden auch eine ausgiebige Literaturrecherche $\mathrm{zu}$ den Schweizer Gesetzesgrundlagen hinsichtlich des kantonalen Gesundheitsgesetzes (Meldepflicht-/recht), des Gewaltschutzgesetzes und des Strafrechts (Offizial-/ Antragsdelikte). Zusätzliche umfassende Praxiseinblicke konnten im Rahmen einer polizeilichen Großkontrolle der Kantonspolizei Zürich gewonnen werden.

\section{Ergebnisse der Ist-Analyse}

\section{Strukturelle Abläufe auf einer Notfallstation}

Hinsichtlich der Erkennung und Behandlung von Personen nach interpersoneller Gewalt zeigt sich, dass Betroffene entweder selbstständig und aus eigenem Antrieb eine Notfallstation aufsuchen oder die bereits involvierte Polizei kann eine Krankenhauseinweisung vornehmen. Wenn Betroffene selbstständig auf der Notfallstation vorstellig werden, kann es sein, dass der wahre Grund ihrer Einweisung nicht sofort oder evtl. sogar gar nicht eruiert wird. Oftmals entsprechen die von Betroffenen gemachten Schilderungen nicht dem tatsächlichem Ereignishergang. Es kommt aber auch nicht selten vor, dass die Angehörigen den Vorfall stellvertretend für die betroffene Person sehr detailliert oder nur ganz grob kursorisch beschreiben und dabei kaum von der Seite des Betroffenen weichen. Das Krankenhauspersonal ist in solchen Situationen oftmals überfordert und erkennt u. U. die „red flags", die auf physische und/oder psychische Gewalt hinweisen, nicht.

Für die von Gewalt betroffenen Personen stellt dies eine absolute Ausnahmesituation dar und ist somit als solche Ausnahmesituation zu behandeln. Die zentrale Herausforderung für sämtliches betreuendes Fachpersonal besteht nun darin, diese Ausnahmesituation von Routinesituationen zu unterscheiden. Somit ist auch das Pflegepersonal gefordert, die Folgen einer Gewalttat richtig zu erkennen, um gezielt darauf $\mathrm{zu}$ reagieren und den Sachverhalt mit der entsprechenden Sorgfalt herauszufinden. Die Angaben zum Sachverhalt erleichtern es den Untersuchenden, die korrekte Untersuchung und Erhebung von Befunden anzugehen, um später über gerichtsverwertbare Beweismittel verfügen zu können. Wird dieser wichtige Triage-Punkt verpasst oder nicht erkannt, gehen evtl. zu sichernde Spuren oder Befunde sehr schnell verloren und sind auch später nicht mehr zu rekonstruieren [3]. Die Ist-Analyse der Abläufe hat ein klares Risiko ergeben. Ebenso wichtig ist die weitere professionelle Betreuung betroffener Personen sowohl in medizinischer als auch in psychologischer Hinsicht. Oft wird es unterlassen, dem gewaltbetroffenen Menschen weiterführende Hilfe anzubieten. Diese kann darin bestehen, dem Betroffenen oder deren Angehörigen Informationen zu bestimmten Hilfsinstitutionen, wie beispielsweise den Opferhilfestellen, zur Verfügung zu stellen. Betroffene Personen sollten zudem über die Möglichkeit, eine Strafanzeige zu stellen, informiert werden, soweit die aktuelle Situation und der Gesundheitszustand des Betroffenen dies erlauben.

Fazit. Die Ist-Analyse der strukturellen Abläufe zeigt Probleme im komplexen Zusammenspiel zwischen medizinischen, rechtlichen und forensischen Aspekten auf. Es ist zwingend nötig, dass eine professionell und speziell auf ebendiese Ausnahmesituationen geschulte Fachperson jederzeit beigezogen werden kann, um die bestehende Lücke zu schließen.

\section{Schnittstellenmanagement}

Es ergibt sich folgendes Bild: Wenn die betroffene Person nach dem Gewaltereignis zuerst die Polizei aufsucht, wird ihr dort ein Merkblatt ausgehändigt. Da die Polizei eine Informationspflicht zu erfüllen hat, muss sie das Opfer der kantonalen Opferhilfestelle mit dem Namen und der Adresse melden. Die Beratungsstelle wird anschließend mit der von Gewalt betroffenen Person Kontakt aufnehmen und sie über ihre Möglichkeiten und Hilfeleistungen informieren. Eine solche Beratung kann auch abgelehnt werden. Die Polizei kann dann in bestimmten Fällen eine rechtsmedizinische Untersuchung veranlassen.

Fazit. Die Wahrscheinlichkeit der Ablehnung einer fachlichen Beratung oder anderweitiger externer Kontaktaufnahme durch die von Gewalt betroffene Person ist eher hoch. Als mögliche Ursache wird die emotional belastende und überfordernde Situation der betroffenen Person genannt. Das gilt speziell und in erhöhtem Maß dann, wenn nicht explizit und professionell auf die Situation der betroffenen Person eingegangen und entsprechend gehandelt bzw. reagiert wird, sei es wegen fehlendem Fachwissen oder schlicht aufgrund fehlender organisatorischer Ressourcen. Professionelles, fachlich korrektes Handeln ist auf den Notfallstationen kaum durchführbar. Aufgrund des andauernden Zeit- und Personalmangels können die oben aufgeführten Anforderungen sehr oft nicht erfüllt werden. Die entsprechende Ausbil- 
Rechtsmedizin 2020 · 30:311-317 https://doi.org/10.1007/s00194-020-00423-y

(c) Der/die Autor(en) 2020

V. Hofer · J. Galli $\cdot$ M. J. Thali $\cdot$ R. M. Martinez

\section{„Flying Forensic Nursing" in der Versorgung und Beratung von Gewaltbetroffenen. Auswertung erster Erfahrungen mit diesem neuen Konzept}

\section{Zusammenfassung}

Hintergrund. "Forensic Nursing" als eine

Spezialisierung des Pflegeberufs hat

sich in der Schweiz zwar mittlerweile in

einzelnen Institutionen durchgesetzt und

wird schrittweise in den Krankenhausalltag

implementiert. Doch längst nicht alle

Institutionen beschäftigen forensisch spezia-

lisierte Pflegefachpersonen. Somit kann ein uneingeschränktes Dienstleistungsangebot,

z. B. ereignisnahe körperliche Untersuchungen nach Gewalt oder Untersuchungen bei V.a. Fahrunfähigkeit, nicht aufrechterhalten werden.

Ziel der Arbeit. Grundlage der vorliegenden Studie ist die Abschlussarbeit einer Certificateof-Advanced-Studies(CAS)-Absolventin im Fachbereich "Forensic Nursing" zum Thema „Flying Forensic Nurse“ [1]. Die Studienergebnisse und die praktischen Erfahrungen einer realen Flying Forensic Nurse sollen die dort getroffenen Schlussfolgerungen ergänzen und das Konzept des Flying Forensic Nursing weiterverfeinern. Damit könnten sich in
Zukunft neue Perspektiven in der modernen Versorgung von Gewaltbetroffenen ergeben. Material und Methode. Eine systematische Auswertung der Istsituation in der Schweiz wurde vorgenommen. In der Projektarbeit waren die formellen und organisatorischen Möglichkeiten der Einbindung einer Flying Forensic Nurse in der Schweiz evaluiert und dargelegt worden. Im Rahmen der Evaluation organisatorischer Aspekte musste zunächst z. B. eruiert werden, ob es sinnvoll ist, dass eine Forensic Nurse "on demand" zur Verfügung steht. Weiterhin galt es, die formellen Rahmenbedingungen, d. h. Anforderungen an eine Forensic Nurse, die Gestaltung eines Aufgabenportfolios und dessen Stellenwert im Kontext rechtlicher Anforderungen, abzuklären.

Ergebnisse. Die Istsituation ist für alle Beteiligten und die involvierten Institutionen als unbefriedigend zu bezeichnen und bedarf der Verbesserung. Zur professionellen, nachhaltigen und einheitlichen Lösung bestehender Lücken in der forensisch-medi- zinischen Versorgung von gewaltbetroffenen Personen kann die dedizierte Einbindung einer Flying Forensic Nurse beitragen. Dieser Ansatz sieht vor, dass forensisch ausgebildete Pflegefachpersonen entweder an ihrem bestehenden Arbeitsort oder auf Abruf einbezogen werden.

Schlussfolgerungen. Die Einbeziehung einer Flying Forensic Nurse in die Untersuchung, Befunderhebung und allfällige Spurensicherung bei Gewaltdelikten ist sinnvoll. Einige Aspekte bedürfen der vorherigen Abklärung. Beispielsweise muss das Anstellungsverhältnis zwischen der Flying Forensic Nurse und dem Auftraggeber klar geregelt werden. Hierzu gehören die Lohn- und Versicherungsverhältnisse sowie die Frage der Qualitätssicherung durch eine definierte Stelle. Diese Aufgaben sollten aber in naher Zukunft lösbar sein.

Schlüsselwörter

Opfer von Gewalt · Körperliche Untersuchung $\cdot$ Dokumentation $\cdot$ Interdisziplinäre Kommunikation · Forensisches Netzwerk

\section{Flying forensic nursing in the care and counselling of victims of violence. Evaluation of first experiences with this new concept}

\section{Abstract}

Background. Forensic nursing is meanwhile an established specialization of the nursing profession in some institutions in Switzerland and is gradually being implemented in hospital routines; however, not all institutions in Switzerland employ forensically specialized nurses. This means that an unlimited range of services offered, for example physical examinations following assault or examinations in suspected impairment of driving capability, cannot be maintained.

Objective. This study was based on the final thesis for a certificate of advanced studies (CAS) in the discipline of forensic nursing on the topic flying forensic nurse. The study results and practical experience of a real flying Forensic Nurse aim to supplement the conclusions of the thesis and further refine the concept of the flying forensic nurse. This could open up new perspectives in the modern care of victims of violence in the future.
Material and methods. A systematic evaluation of various aspects of the current situation in Switzerland was carried out. In the project work the formal and organizational possibilities of integrating a flying forensic nurse in Switzerland were evaluated and presented. The focus of the evaluation was initially placed on the organizational aspects, e.g. it had to be determined whether it makes sense for a forensic nurse to be available on demand. Furthermore, it was necessary to clarify the formal framework conditions, i.e. the requirements for a flying forensic nurse, the structure of a possible task portfolio and its value in the context of legal requirements. Results. The current situation is unsatisfactory for all persons and institutions involved and urgently needs to be improved. The dedicated involvement of a flying forensic nurse can contribute to a professional, sustainable and standardized solution of existing gaps in the forensic medical care of people affected by violence. The aim of this approach is that forensically trained nursing personnel are involved either at their existing place of work or on demand.

Conclusion. The inclusion of a flying forensic nurse in the investigation, reporting and possible securing of evidence in cases of violent crime makes sense. Some aspects need to be clarified. For example, the employment relationship between the flying forensic nurse and the client must be clearly regulated. This includes the salary and insurance relationships as well as the question of quality assurance by a defined body; however, these should be solvable tasks for the near future.

\section{Keywords}

Crime victims - Physical examination · Documentation - Interdisciplinary communication . Forensic network 
dung sowie das erforderliche Wissen über die rechtlichen Grundlagen und über die Hilfsangebote (auch in der eigenen Organisationseinheit) versinken oftmals im hektischen Routinegeschäft der Notfallstation.

\section{Erfüllung forensischer Aufgaben}

Die Erfüllung forensischer Aufgaben wie Dokumentation der Befunde und Sicherung biologischer Spuren auf der Notfallstation weist die größten Lücken auf.

Wenn Personen nach interpersoneller Gewalt direkt, ohne Einweisung einer entsprechenden Institution, in ein Krankenhaus gehen, wird in der Notfallstation eine Triage mit einer kurzen schriftlichen Ersteinschätzung zuerst durch eine Pflegefachfrau vorgenommen und danach eine körperliche Untersuchung des Betroffenen durch einen Arzt durchgeführt. Eine solche Untersuchung durch einen Krankenhausarzt ist nicht mit einer rechtsmedizinischen Untersuchung gleichzustellen. Die Ärzte auf der Notfallstation dokumentieren die vorhandenen Verletzungen meist nur grob kursorisch, stellen Differenzialdiagnosen und ordnen medizinische bzw. diagnostische Maßnahmen an. Dabei werden Befunde wie Blutergüsse oder kleinste punktförmige Einblutungen im Gesichtsbereich und ihre Bedeutung selten erkannt, geschweige denn entsprechend detailliert dokumentiert. Auch Graß und Rothschild stellten bereits fest:

Im Gegensatz zur klassischen ärztlichen Ausrichtung auf eine effiziente Diagnostik und beste Therapie spielen für rechtliche Fragestellungen spezifische Verletzungsbefunde und deren sorgfältige Dokumentation eine Rolle, denen oft keine therapeutische Relevanz zukommt; Stichwort „Bagatellverletzungen“, z. B. kleine Kratzer in der Hals- und Gesichtshaut bei einem Opfer, das angibt, den Mund zugehalten bekommen zu haben und gewürgt worden zu sein. (Graß und Rothschild [4])

Die klinisch-ärztlichen Berichte unterscheiden sich zudem von den rechtsmedizinischen Berichten: Erstere werden in einer fachmedizinischen Sprache verfasst und sind für juristische Fachpersonen nicht oder wenig verständlich. Somit sind diese Dokumentationen meist nicht oder nur eingeschränkt gerichtsverwertbar. Die vollständige und gerichtsverwertbare Untersuchung und Befunddokumentation erfordern ein gut strukturiertes Dokumentationsinstrument, das aber auf den Notfallstationen oftmals nicht vorhanden ist. Eine Körperschemavorlage, in dem die Verletzungen von Hand und in verschiedenen Farbmustern eingezeichnet werden, fehlt meist. Auch die fotografische Dokumentation wird erfahrungsgemäß oft unterlassen. Genau dieser spezifische Prozessschritt hat jedoch einen enorm hohen Stellenwert, um die forensisch relevanten Befunde festzuhalten. Wenn doch fotografisch dokumentiert wird, sind diese Bilder oft nicht in einer ausreichenden Qualität erstellt bzw. gesichert worden. Das Erstellen eines Gesamtübersichtsbildes des Patienten wird meist ganz unterlassen, und sehr oft werden nur Detailaufnahmen der vorhandenen Verletzungen der betreffenden Körperregion angefertigt. Aufgrund schlechter Bildqualität, blendender resp. farbverfälschender Effekte durch Blitz- oder Kunstlicht sind die Bilder für rechtsmedizinische $\mathrm{Be}$ lange oft unbrauchbar. Die eindeutige Identifizierung der betroffenen Person wird unterlassen; Größenangaben von Verletzungsmustern auf fotografischen Aufnahmen mithilfe eines farbechten Winkelmeters und die genaue Bezeichnung der betroffenen Körperregionen fehlen. Dies alles trägt dazu bei, dass diese Dokumentationen letztendlich nicht rechtsmedizinisch auswertbar bzw. gerichtsverwertbar sind.

Fazit. Die Ist-Analyse ergibt also eine unbefriedigende Situation. Die korrekte Spurensicherung oder zumindest der erforderliche Spurenschutz in zunächst unklaren Fällen oder Angaben zum Ereignis fehlen bzw. werden im klinischen Setting oft mangels Kenntnis und auch aus Überforderung der beteiligten medizinischen Fachpersonen unterlassen. Nachteilig ist außerdem die Tatsache, dass eine Spurensicherung meist nur dann erfolgt, wenn die Polizei und/oder die Staatsanwaltschaft bereits involviert ist und einen entsprechenden Auftrag er- teilt hat. Geschieht dies nicht, werden Verletzungen vom medizinischem Fachpersonal meist sofort gereinigt, chirurgisch versorgt und damit zwangsläufig evtl. vorhandene wichtige und aussagekräftige Spuren unwiederbringlich zerstört. Weitergehend wird sehr oft die professionelle und korrekte DNA-Sicherung in Unkenntnis der tatsächlich vorliegenden Situation und aufgrund von fehlendem geeigneten Entnahmematerial unterlassen. Dies beinhaltet ebenso die Sicherstellung der Kleidung der gewaltbetroffenen Menschen. Die Kleidung wird in der Hektik einer Akutsituation ausgezogen und/oder zerschnitten und ohne korrekte Sicherung evtl. Spuren in einem Patienteneffektensack deponiert [5].

\section{Rechtliche Aspekte und ihre Auswirkungen auf die Strukturen einer Notfallstation}

Hier zeigen sich weitere Verunsicherungen des Krankenhauspersonals auf. Was sind Handlungsfelder und Befugnisse, aber auch klar definierte Grenzen des Pflegepersonals in Bezug auf die Untersuchung von und Befunderhebung bei gewaltbetroffenen Personen, die die Notfallstation freiwillig aufsuchen und eine medizinische Versorgung sowie umfassende Dokumentation von Verletzungen ohne eine Strafanzeige wünschen? Die erste Diskrepanz entsteht bereits bei der Betrachtung der Anstellungsverhältnisse einer forensisch ausgebildeten Pflegefachperson: Gemäß Arbeitgeber sei sie primär als Pflegefachperson angestellt und, falls keine klare Vereinbarung zwischen dem Arbeitgeber (Krankenhaus) und der Pflegefachperson in Bezug auf die Erbringung der Zusatzleistungen als Forensic Nurse besteht, gibt es vom Arbeitgeber auch keinen rechtsverbindlichen Auftrag. Dazu sagt der Gesetzgeber Folgendes:

„Art. 251 StPO [6]

1. Die Untersuchung einer Person umfasst die Untersuchung ihres körperlichen oder geistigen Zustands.

2. Die beschuldigte Person kann untersucht werden, um:

a. den Sachverhalt festzustellen; 
b. abzuklären, ob sie schuld-, verhandlungs- und hafterstehungsfähig ist.

3. Eingriffe in die körperliche Integrität der beschuldigten Person können angeordnet werden, wenn sie weder besondere Schmerzen bereiten noch die Gesundheit gefährden.

4. Gegenüber einer nicht beschuldigten Person sind Untersuchungen und Eingriffe in die körperliche Integrität gegen ihren Willen zudem nur zulässig, wenn sie unerlässlich sind, um eine Straftat nach den Artikeln 111-113, 122, 124, 140, 184, $185,187,189,190$ oder 191 StGB [7] aufzuklären.“

Von Gewalt betroffene Menschen werden häufig von der Polizei an die Notfallstation verwiesen. Dies erfolgt mitunter einfach mit den Worten: „Gehen Sie in die Notfallstation, damit Sie einen medizinischen Bericht für die Akten erhalten. "In dieser Situation ist es für das Pflegepersonal oft schwierig herauszufinden, ob schon irgendwelche Dokumentationen und/oder Fotos durch die Polizei angefertigt wurden. Zudem hat das Krankenhauspersonal keine Angaben über schon getätigte Gewaltschutzmaßnahmen oder weitere Vorgehensweisen, die die Polizei veranlasst hat. Die Betroffenen fragen in einem Krankenhaus das Pflegepersonal, was die nächsten Schritte bzw. Konsequenzen des polizeilichen oder rechtsmedizinischen Einbezugs für sie bedeuten würden. Um eine professionelle Anamnese zu erheben, die für eine vollständige und gerichtsverwertbare Dokumentation unerlässlich ist, ist ein vorheriger Informationsaustausch mit der Polizei bzw. mit den überweisenden Stellen von großer Bedeutung. Doch nicht zuletzt wegen der Einschränkungen im Datenschutzgesetz ist dieser Austausch kaum möglich, es sei denn, dass eine entsprechende Entbindung vom Berufsgeheimnis (Art. 321 StGB) bereits vorliegt.

\section{Handhabung von Asservaten auf der Notfallstation}

Die Handhabung von Asservaten wie Blut- und Urinproben auf der Not- fallstation stellt ein komplexes interdisziplinäres Zusammenspiel dar. Auf Notfallstationen ist es an der Tagesordnung, dass die Polizei mit den Personen, die sie zwecks ärztlicher Untersuchung bei V.a. Beeinträchtigung, z. B. Fahren in angetrunkenem Zustand unter Drogeneinfluss oder unter Medikamentenwirkungen, zuführt, meist sehr lange Wartezeiten in Kauf nehmen muss. Diese Untersuchungen werden von ärztlicher Seite als „nichtprioritär“ angesehen. Viele Ärzte widmen sich zuerst den „richtigen Notfällen“ und erledigen die Untersuchung der zugeführten Person irgendwann später oder schieben sie irgendwo schnell dazwischen. Zudem sind das Konzept einer Fahrfähigkeitsuntersuchung (FFU) und deren Konsequenzen nicht bekannt bzw. werden oft unterschätzt (z. B. die Bedeutung der Leistungsreserve im Straßenverkehr sowie die korrekte Durchführung einzelner Funktionsprüfungen).

In der Literatur gibt es nur einzelne Studien zur Qualität der FFU durch Polizei/Ärzte [8]. Die retrospektiven Studien $[8,9]$ zeigen, dass:

- Ärzte in den Krankenhäusern die Fahrunfähigkeit weit weniger gut als geschulte Polizisten erkennen,

- weder die Polizei noch der beigezogene Arzt die Ursache von Fahrunfähigkeit (Alkohol und/oder andere Substanzen) sicher erkennen können,

- bei der FFU der zeitnahen Beurteilung durch den Polizisten höheres Gewicht eingeräumt werden sollte.

Fazit. Eine forensisch und medizinisch geschulte Fachperson (Forensic Nurse) kann im typischen Setting aussagekräftigere Ergebnisse einer FFU erzielen als der Arzt. Eine ereignisnahe und vollständige FFU, einschließlich Blutund Urinasservierung, kann durch eine dafür ausgebildete Forensic Nurse gewährleistet werden. Dazu sind aktuell jedoch entsprechende Anpassungen in der Gesetzgebung notwendig, damit eine gerichtsverwertbare Durchführung einer FFU durch eine Forensic Nurse vorgenommen und rechtlich akzeptiert werden kann. Die Zusammenarbeit zwischen Forensic Nurses, klinisch tätigen
Ärzten und rechtsmedizinischen Instituten ist die zentrale Voraussetzung für eine geregelte, effiziente, nachhaltige und gerichtsverwertbare Umsetzung solcher genannten Synergien in Fällen von forensischer Relevanz, u. a. bei der FFU oder bei den Untersuchungen nach interpersoneller Gewalt [10].

\section{Resümee}

Die Ist-Analysen aller Teilaspekte zeigen, dass die Situation für alle beteiligten Personen und Institutionen zum augenblicklichen Zeitpunkt nur als unbefriedigend zu bezeichnen ist und dringend einer Verbesserung bedarf. Der Bedarf an einer professionellen, nachhaltigen und einheitlichen Lösung zur Abwicklung diesbezüglicher Abläufe ist bei allen beteiligten Schnittstellen und v.a. bei den von Gewalt betroffenen Personen groß.

\section{Diskussion}

Zu Beginn der Diskussion sollte klar gesagt werden, dass die vorliegende Studie Limitierungen aufweist. In Ermangelung einer flächendeckenden Versorgung von Gewaltopfern unter Hinzuziehung einer Flying Forensic Nurse muss auf Einzelerfahrungen zurückgegriffen werden. Sollte es gelingen, weitere Forensic Nurses in den Dienst dieser für die klinische Rechtsmedizin so wichtigen Disziplin einzubinden, können größere Studien ins Leben gerufen werden, die noch zuverlässigere Aussagen über eine mögliche Ausgestaltung der neuen Disziplin Flying Forensic Nursing erlauben. Immerhin ist es in der vorliegenden Studie gelungen, die Istsituation aller Teilaspekte in der Schweiz zu ermitteln und systematisch auszuwerten. So kann festgestellt werden, dass die Kommunikation über definierte Schnittstellen zwischen den involvierten Institutionen unkoordiniert ist, womit wichtige Informationen unvollständig oder nicht beim Adressaten ankommen. Betroffene werden nach einem Gewaltereignis in einer manifesten, emotional aufgeladenen Ausnahmesituation von einer Institution zur anderen geschickt, oftmals ohne einen schriftlichen Bericht oder ohne generell in einer solchen Situation adäquate Informatio- 
nen erhalten zu haben. Dieses Vorgehen verursacht eine enorme Unsicherheit bei allen Beteiligten, bedingt meist zusätzliche, nachträgliche und damit zeitintensive Arbeitsschritte und wird somit zum Kostentreiber „par excellence“. So entstehen zwangsläufig Fehler, z.B. durch unvollständiges oder fehlerhaftes Ausfüllen des Untersuchungsprotokolls, nicht zuletzt durch fehlendes Prozessund Qualität-Monitoring. Es erweist sich unter den gegebenen Umständen z.B. als fast unmöglich, eine einheitliche, für alle involvierten Institutionen generisch auswertbare, professionelle, gerichtsverwertbare Dokumentation und Untersuchung der gewaltbetroffenen Menschen sicherzustellen.

Anders zeigt sich die Situation der forensischen Pflege in den USA: Dort ist die professionelle Einbindung von forensisch ausgebildeten Pflegefachpersonen in allen Staaten vollumfänglich etabliert. Es gibt im Bereich Forensic Nursing verschiedene Spezialisierungen wie beispielsweise „sexual assault nurse examiner“ (SANE) oder „forensic nurse examiner" (FNE). Auch die Kompetenzen einer Forensic Nurse sind dort weitestgehend geregelt. $\mathrm{Ob}$ ein bestimmter Standard oder eine bestimmte Kompetenz gilt, hängt von den Gesamtumständen ab. Forensic Nurses, die innerhalb dieser bestimmten Rolle, Bevölkerungsgruppe und Fachrichtung tätig sind, können Entwicklungsmöglichkeiten und Verbesserungen erkennen und weiterverfolgen, indem sie ihre Leistungen anhand dieser Elemente kontinuierlich evaluieren [11]. Eine Forensic Nurse in den USA:

- sammelt in einem systematischen und fortlaufenden Prozess Daten zu körperlichen Befunden und Verhaltensweisen, wobei der Schwerpunkt auf der Pflege der Patienten und der Identifizierung der medizinischrechtlichen Implikationen dieser Befunde liegt;

- setzt Prioritäten für die Datenerhebung auf der Grundlage des unmittelbaren Zustands des Patienten, der Bedürfnisse des Patienten oder der Situation und der Sicherung rechtlicher Beweise;
- verwendet geeignete evidenzbasierte Beurteilungstechniken und -instrumente bei der Datenerhebung;

- dokumentiert relevante Daten und Befunde;

- wendet analytische Modelle und Problemlösungsinstrumente in der Forensic-Nursing-Praxis an;

- analysiert verfügbare Daten, Informationen und situationsrelevantes Wissen, um Muster und Abweichungen zu erkennen;

- identifiziert Hindernisse für eine effektive Kommunikation und nimmt entsprechende Anpassungen vor;

- beachtet ethische, rechtliche und datenschutzrechtliche Richtlinien und Grundsätze bei der Sammlung, Pflege, Nutzung und Verbreitung von Daten und Informationen.

Auch die Finanzierung der erbrachten Leistungen durch eine forensisch ausgebildete Pflegefachperson ist in den USA weitestgehend durch verschiedene Finanzierungsprogramme geregelt [12], z.B. international geregelte Abrechnungs- und Kodierungspraktiken in der Internationalen statistischen Klassifikation der Krankheiten und verwandter Gesundheitsprobleme (ICD-10). Um sowohl Versicherungsgesellschaften als auch Opferentschädigungskassen Rechnungen zu stellen, muss das SANE-Programm u. U. Einzelverbindungsnachweise unter Verwendung der Standardabrechnungspraktiken im Gesundheitswesen vorlegen. Es werden aber auch andere Finanzierungsprogramme angewendet, beispielsweise geregelte Honorare für Sachverständige oder krankenhausfinanzierte Programme. Diese geregelten Strukturen in den USA ermöglichen es den forensisch ausgebildeten Pflegefachpersonen, ihre Fähigkeiten und ihr Wissen in Fällen von forensischer Relevanz zielgerichtet einzusetzen und somit einen hohen Stellenwert im komplexen medizinisch-rechtlichen System zu sichern.

\section{Ausblick}

Forensisch ausgebildete Pflegefachpersonen arbeiten weiter daran, die Situation in der Schweiz nach amerikanischem
Vorbild zu verbessern. So soll ein übergeordnetes Tätigkeitsprofil für Forensic Nurses in der Schweiz erarbeitet werden. Auch soll die Qualitätssicherung für die Absolventen und Absolventinnen von Fort- und Weiterbildungen in forensischer Pflege verbessert werden. Dank nationaler und internationaler Vernetzung mit anderen Forensic Nurses, wie z.B. über die Academy of Forensic Nursing (AFN, [13]), die International Association of Forensic Nurses (IAFN, [14]) oder die Swiss Association Forensic Nursing (SAFN, [15]), bleiben die Forensic Nurses immer auf dem aktuellsten Wissensstand.

\section{Fazit für die Praxis}

- Die Rolle einer forensisch ausgebildeten Pflegefachperson in der klinischen Praxis mit forensischer Relevanz wird in der Schweiz immer gefestigter. Zugleich zeigen sich bei der schrittweisen Umsetzung des Forensic Nursing strukturelle und organisatorische Felder mit dringendem Abklärungsbedarf.

- Die bisherigen Implementierungserfahrungen machen deutlich, dass v. a. Gemeinschafts- und Hausarztpraxen, aber auch gynäkologische und pädiatrische Praxen primär von der Einbindung einer Forensic Nurse profitieren würden.

- Das Projekt "Flying Forensic Nursing“, also Forensic Nurse "on demand", wie es beispielsweise in der Zusammenarbeit mit Amtsärzten oder anderen Auftraggebern vorgesehen ist, bedarf tieferer übergeordneter Abklärungen, nicht zuletzt auf politischer und rechtlicher Ebene.

- Nichtsdestotrotz hat das Projekt ganz eindeutig das Potenzial, in der modernen Versorgung und Gewaltprävention deutliche Verbesserungen zu erbringen.

\section{Korrespondenzadresse}

\section{Hofer}

Institut für Rechtsmedizin, Universität Zürich Winterthurerstr. 190/52, 8057 Zürich, Schweiz valeria.hofer@irm.uzh.ch 
Funding. Open access funding provided by University of Zurich

\section{Einhaltung ethischer Richtlinien}

Interessenkonflikt. V. Hofer, J. Galli, M.J. Thali und R.M. Martinez geben an, dass kein Interessenkonflikt besteht.

Für diesen Beitrag wurden von den Autoren keine Studien an Menschen oder Tieren durchgeführt. Für die aufgeführten Studien gelten die jeweils dort angegebenen ethischen Richtlinien.

Open Access. Dieser Artikel wird unter der Creative Commons Namensnennung 4.0 International Lizenz veröffentlicht, welche die Nutzung, Vervielfältigung, Bearbeitung, Verbreitung und Wiedergabe in jeglichem Medium und Format erlaubt, sofern Sie den/die ursprünglichen Autor(en) und die Quelle ordnungsgemäß nennen, einen Link zur Creative Commons Lizenz beifügen und angeben, ob Änderungen vorgenommen wurden.

Die in diesem Artikel enthaltenen Bilder und sonstiges Drittmaterial unterliegen ebenfalls der genannten Creative Commons Lizenz, sofern sich aus der Abbildungslegende nichts anderes ergibt. Sofern das betreffende Material nicht unter der genannten Creative Commons Lizenz steht und die betreffende Handlung nicht nach gesetzlichen Vorschriften erlaubt ist, ist für die oben aufgeführten Weiterverwendungen des Materials die Einwilligung des jeweiligen Rechteinhabers einzuholen.

Weitere Details zur Lizenz entnehmen Sie bitte der Lizenzinformation auf http://creativecommons.org/ licenses/by/4.0/deed.de.

\section{Literatur}

1. J. Galli, "Flying Forensic Nurse," CAS in Forensic Nursing UZH, 2017. www.forensicnursing.ch

2. Mützel E, Helmreich C, Schick S, Saß M, Schöpfer J (2014) Klinisch-forensische Versorgung von Gewaltopfern in Bayern. Rechtsmedizin 24:200

3. Lynch V, Duval J (2011) Forensic nursing science, 2. Aufl. Elsevier, St. Louis, S123 (Chap. 11)

4. Graß H, Rothschild M (2004) Aufgaben und Herausforderungen im Rahmen der medizinischen Betreuung von Opfern häuslicher Gewalt. Rechtsmedizin 14(14):188

5. Wick J (2000) Don't destroy the evidence! AORN J 72(5):807-836. https://doi.org/10.1016/s00012092(06)62013-9

6. Schweizerische Strafprozessordnung, StPO. https://www.admin.ch/opc/de/classifiedcompilation/20052319/index.html

7. Schweizerisches Strafgesetzbuch, StGB

8. Steuer A, Eisenbeiss L, Krämer T (2016) Blood alcohol analysis alone versus comprehensive toxicological analysis-Systematic investigation of missed co-ingested other drugs in suspected alcohol-impaired drivers. Forensic Sci Int 267:52-59

9. Krämer T (2019) Können Polizisten und Ärzte Fahrunfähigkeit und ggf. deren Ursacheerkennen? In: Hardy L, Manfred D (Hrsg) Jahrbuch zum Strassenverkehrsrecht 2019DikeVerlag, 1. Auflage, Zürich/St. Gallen 2019, S225-232
10. Hofer V, Thali MJ, Keller K, Martinez RM (2019) Effizienz durch Synergien - Untersuchung bei Verdacht auf Fahrunfähigkeit durch Forensic Nurses. In: 98. Jahrestagung der Deutschen Gesellschaft für Rechtsmedizin Hamburg

11. International Association of Forensic Nurses, American Nurses Association (2015) Forensic nursing scope and standards. International Association of Forensic Nurses, American Nurses Association, Silver Spring, Maryland

12. „Office of Justice Programs," Office for Victims of Crime SANE program development and operation guide. https://www.ovcttac. gov/saneguide/program-operational-costs-andfunding/financing-your-program/.Zugegriffen:3. Aug. 2020

13. Academy of Forensic Nursing (AFN) www.goafn. org. Zugegriffen:3. Aug. 2020

14. International Association of Forensic Nurses (IAFN) www.forensicnurses.org.Zugegriffen:3. Aug. 2020

15. Swiss Association Forensic Nursing (SAFN) www. forensicnursing.ch.Zugegriffen:3.Aug. 2020
Hier steht eine Anzeige. Springer 